\title{
Distinguishing child abuse fractures from rickets
}

\author{
Alan E. Oestreich ${ }^{1}$ \\ Received: 28 July 2017 / Revised: 6 October 2017 / Accepted: 8 November 2017 / Published online: 24 November 2017 \\ (C) Springer-Verlag GmbH Germany, part of Springer Nature 2017
}

\section{Introduction}

The keystone of distinguishing suspected child abuse on radiographs from manifestations of rickets, including healing rickets, is looking at bones other than that bone suspected of rickets, as well as carefully perusing the bone under suspicion. Understanding the metaphyseal collar and the zone of provisional calcification as part of the normal, non-rachitic, tubular bone metaphyses should help distinguish between rickets and changes related to fracture.

\section{The denialists}

As was strongly proclaimed by an editorial in this journal [1], a great problem exists from those medical professionals who, in testimony and in print, deny abuse as a cause for radiologic abnormalities widely accepted as suggestive of child abuse.

\section{Child abuse vs. rickets}

Child abuse denialists often claim that radiographic findings generally recognized by pediatric radiologists as having high specificity for abusive trauma are instead a consequence of rickets, including healing rickets [2]. In particular, bucket handle fractures (classic metaphyseal lesions) at metaphyses are declared to be identical in pattern to healing rickets [2]. I

Alan E. Oestreich

alan.oestreich@cchmc.org

1 Department of Radiology ML5031,

Cincinnati Children's Hospital Medical Center,

3333 Burnet Ave., Cincinnati, OH 45229-3039, USA demonstrate by reviewing the details of these different patterns that this is an incorrect declaration. Additionally, concavity of the distal ulna metaphysis alone is not a sign of rickets [3]. Rickets is radiologically distinct from classic metaphyseal fractures (see also [4]).

Among the claims put forth by denialists is that the appearance of healing rickets may be mistaken for a bucket handle type of classic metaphyseal lesion seen in child abuse [2]. I contend that although there may be a superficial resemblance between the two entities, namely that both occur in the metaphyses, no other features of rickets are present with child abuse fractures. In particular, the metaphyseal collar (of LavalJeantet et al. [5]) is preserved at the end of the bone in the absence of rickets. Moreover, the metaphyseal collar and the zone of provisional calcification are preserved at all other bones locally and throughout the child. Figure 1 demonstrates these entities absent in rickets. Figure 2 demonstrates them preserved in nonrachitic abuse. It should be noted that the sites of fastest growth - especially where the end of the bone is straight, such as the distal radius, distal ulna, the metaphyses at the knee, and the distal tibia and fibula - are the most sensitive sites for detecting rickets, including evaluation of zones of provisional calcification and preservation of the metaphyseal collar. At some sites of slower growth, the zone of provisional calcification may still appear present in rickets in spite of rachitic change elsewhere.

Critical to understanding and recognizing rickets at the metaphyseal ends of tubular bones, particularly the distal radius and ulna and the distal femur and tibia, is the normal metaphyseal collar configuration described by M. LavalJeantet, MD, some 50 years ago [5]. This longitudinally straight metaphyseal margin, generally $1-3 \mathrm{~mm}$ in length throughout childhood, is clearly distinct from the curved diametaphyseal margins that continue on to the diaphysis (Fig. 3). Incidentally, the change from straight metaphyseal 


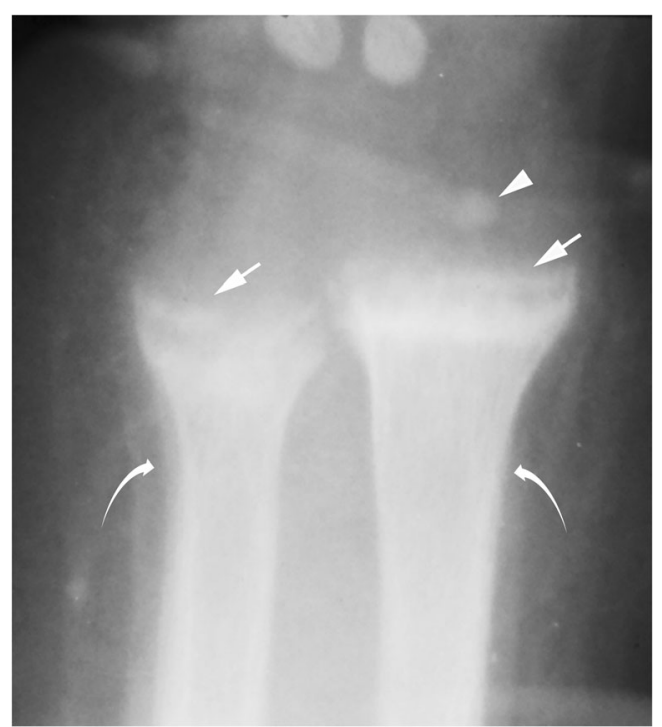

Fig. 1 Anteroposterior radiograph of the wrist shows early remineralization of the zone of provisional calcification (straight arrows) at the distal radius and ulna, seen as a coarse unsharply defined calcific density paralleling the entire already ossified metaphyseal bone that predated the rickets in a 6-month-old girl. Note that the zone of provisional calcification of the distal radius epiphysis (arrowhead) is not calcified and therefore not well defined. Moreover, the cortices of the radius and ulna (curved arrows) are still unsharply defined ("washed out") from the girl's secondary hyperparathyroidism

collar to curved diametaphyseal margin (as shown in Fig. 3) should not be mistaken for a buckle fracture. Because of the abnormal lack of mineralization of physeal cartilage at and beyond the zone of provisional cartilage in rickets, the collar

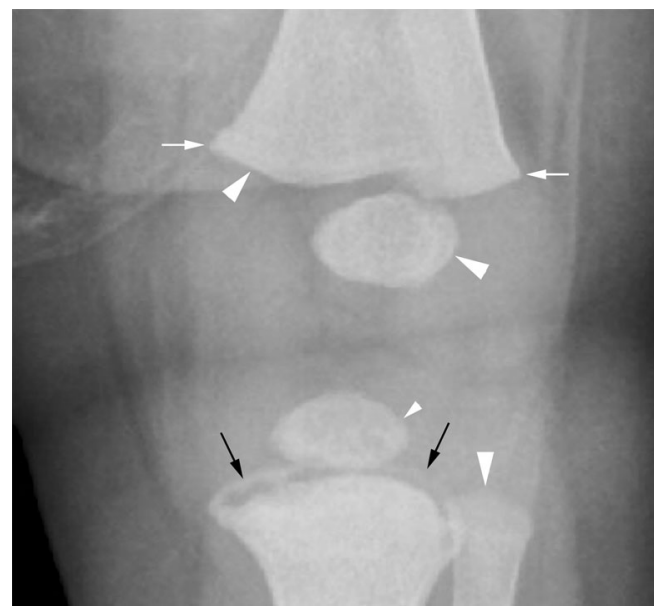

Fig. 2 Anteroposterior radiograph of the left knee shows a bucket handle fracture fragment (black arrows) from child abuse (in a 3-month-old girl who also had a suspicious fracture of a humerus diaphysis). The indicated bucket handle fragment is more sharply defined than the healing zone of provisional calcification of Fig. 1. It is very important to note that there is no other sign of rickets. Well-defined zones of provisional calcification are seen at the distal femoral metaphysis and epiphysis, and the proximal fibular metaphysis and the proximal tibial epiphysis (arrowheads), and a normal metaphyseal collar is noted at the medial and lateral distal femur (white arrows)

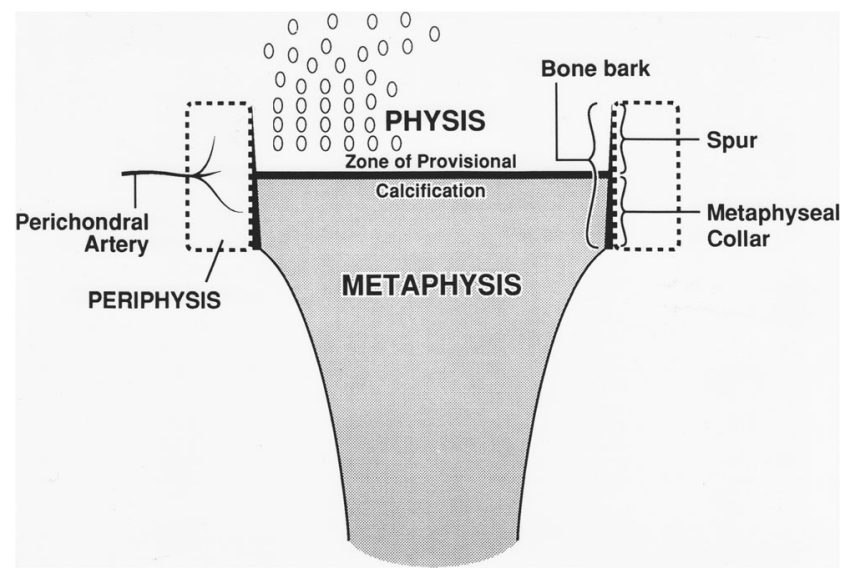

Fig. 3 Diagrammatic representation shows the metaphyseal collar (of Laval-Jeantet et al. [5]), extending from the zone of provisional calcification to a step-off at the beginning of the curved margin of cortex in the diametaphysis. Reprinted with permission from Oestreich AE, Ahmad BS (1992) The periphysis and its effect on the metaphysis: I. definition and normal radiographic pattern. Skeletal Radiol 21:283-286

is then not visible (although a thin bone bark at its outer margins may still be perceived). However, if the collar is preserved, the child does not have rickets. In metaphyseal fractures from abuse, the zone of provisional calcification and collar are maintained. In early healing rickets, the zone of provisional calcification calcifies first, giving a transverse calcification separated from the already ossified metaphysis (as in Fig. 1). This calcification is somewhat different from a bucket handle fracture pattern in that no signs of rickets are found in the region (including no loss of zones of provisional calcification) in abuse. In children with early healing rickets that I have encountered, the transverse dense line of healing zones of provisional calcification has been less sharply defined than bucket handle fracture fragments (compare Fig. 1 with Fig. 2).

\section{Other comments on rickets}

Another recurrent misconception in denialist literature and testimony has been considering the concave distal end of the ulna in itself as evidence of rickets. We have shown in a random review of infants with no other evidence of rickets that $20 \%$ have some degree of concave distal ulna metaphysis (Fig. 4) [3]. An intact Laval-Jeantet collar at the distal end of the ulna as well as the adjacent radius and no loss of calcified zones of provisional calcification are further evidence of the absence of rickets in those children. An example of a case in which normal concave distal ulna is declared rickets [6] exemplifies those other non-rachitic findings. This article, cited in an article by two of the denialists [7], clearly shows intact metaphyseal collars and zones of provisional calcification, not rickets. 


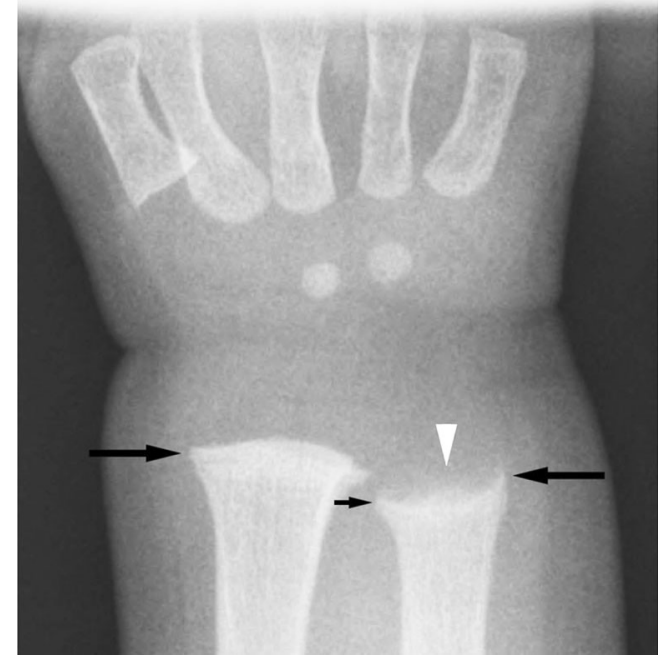

Fig. 4 Anteroposterior wrist radiograph as part of a normal skeletal survey in a 7-month-old boy. Moderate distal concavity of the distal end of the ossified ulna metaphysis (arrowhead) is normal. Arrows point to the intact normal metaphyseal collar of the distal radius and ulna. Zones of provisional calcification are ossified at the distal radius and ulna, around the carpal bones, and at the proximal margins of the metacarpal. Findings indicate the absence of rickets. The tubular bones also show no evidence of secondary hyperparathyroidism, further supporting the absence of rickets. Reprinted with permission [3]

Another finding to support a diagnosis of healing rickets is seeing the reappearance of the zone of provisional calcification around secondary growth centers, separated from the previously ossified epiphysis, as shown in Fig. 5 of this piece, as well as in Fig. 5 of reference [8]. This would not be expected with a bucket handle fracture.

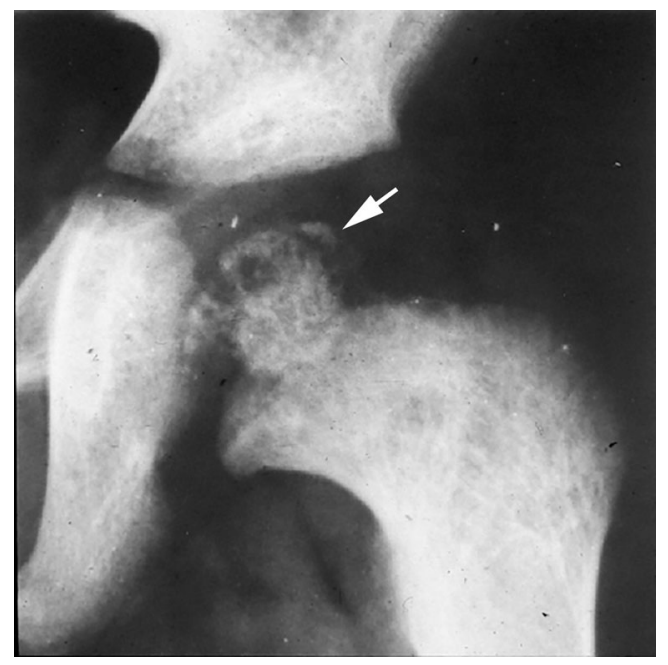

Fig. 5 Anteroposterioor radiograph of the left hip of a young child of unknown age and gender from Paris, France, recovering from nutritional rickets at the end of World War II. The arrow points to the newly calcified zone of provisional calcification surrounding the already ossified proximal femoral epiphysis. (Image courtesy of Professor M. LavalJeantet, MD, Paris. Reprinted with permission [8])
Most children with most types of rickets manifest findings of secondary hyperparathyroidism as well [9]. Findings of hyperparathyroidism include loss of lamina dura around unerupted teeth, washing out (increased porosity) of tubular bone cortices and coarsened trabeculae within tubular bones. The lack of these findings decreases the likelihood of rickets being present.

\section{Summary}

If there is suspicion of rickets at the end of a tubular bone one must carefully evaluate all of the imaged bones. If the metaphyseal collars of Laval-Jeantet are intact and if the zones of provisional calcification are calcified, then the child does not have rickets. Rickets is systemic and symmetrical; classic metaphyseal fractures are not. Patterns of fracture considered to be of high specificity for child abuse are distinctly different from healing rickets and this should not be denied.

Acknowledgments Based on a talk at the European Society of Paediatric Radiology in Davos, Switzerland, on June 1, 2017.

\section{Compliance with ethical standards}

Conflicts of interest None

\section{References}

1. Strouse PJ (2016) Child abuse: we have problems. Pediatr Radiol 46: $587-590$

2. Ayoub DM, Hyman C, Cohen M et al (2014) A critical review of the classic metaphyseal lesion: traumatic or metabolic? AJR Am J Roentgenol 202:185-196

3. Oestreich $\mathrm{AE}$ (2015) Concave distal end of ulna metaphysis alone is not a sign of rickets. Pediatr Radiol 45:998-1000

4. Kepron C, Pollanen MS (2015) Rickets or abuse? A histologic comparison of rickets and child abuse-related fractures. Forensic Sci Med Pathol 11:78-87

5. Laval-Jeantet M, Balmain N, Juster M et al (1968) Les rapports de la virole périchondrale et du cartilage en croissance normale et pathologique [Relations of the perichondral ring to the cartilage in normal and in pathological growth]. Ann Radiol 11:327-335

6. Russell JGB, Hill LF (1974) True fetal rickets. Brit. J Radiol 47:732734

7. Paterson CR, Ayoub D (2015) Congenital rickets due to vitamin D deficiency in the mothers. Clin Nutr 34:793-798

8. Oestreich AE (2004) The acrophysis: a unifying concept for understanding enchondral bone growth and its disorders. II. Abnormal growth. Skelet Radiol 33:119-128

9. Holm I, Perez-Rosello JM (2015) Disorders of calcium and phosphorus metabolism. In: Kleinman PK (ed) Diagnostic imaging of child abuse, $3^{\text {rd }}$ edn. Cambridge University Press, Cambridge, pp 240-253 